\title{
Investigation into the cause of mortality in 49 cases of idiopathic inflammatory myopathy: A single center study
}

\author{
YIZHI XIAO, XIAOXIA ZUO, YUNHUI YOU, HUI LUO, LIPING DUAN, WEIRU ZHANG, \\ YISHA LI, YANLI XIE, YAOU ZHOU, WANGBIN NING, TONG LI, SIJIA LIU, \\ HONGLIN ZHU, YING JIANG, SIYAO WU and HONGJUN ZHAO
}

Department of Rheumatology and Immunology, Xiangya Hospital, Central South University, Changsha, Hunan 410008, P.R. China

Received May 15, 2015; Accepted December 17, 2015

DOI: $10.3892 /$ etm.2016.3006

\begin{abstract}
Idiopathic inflammatory myopathy (IIM) is an autoimmune disease characterized by chronic muscle weakness and myositis with unknown etiology. IIM may affect the function of multiple organs and has a poor prognosis. In the present study, the causes of mortality in patients with IIM admitted to the Xiangya Hospital during the last 14 years were investigated. The investigation included an analysis of frequent causes of IIM, and of infections and associated complications. A cohort study was conducted on 676 patients with IIM that were admitted to Xiangya Hospital from January, 2001 to January, 2015. There were 49 patient mortalities (7.2\% of the total cases), of which 34 mortalities were infection-associated and 15 were not infection-associated. The proportion of infection-associated IIM mortalities had increased since 2001. Of the 34 infection-associated mortalities, 31 cases $(63.3 \%)$ were of fungal and bacterial infections, most frequently infecting the lungs and the blood. Klebsiella pneumoniae and Acinetobacter baumannii were the most commonly isolated pathogens, and co-infection with the two pathogens was observed in the majority of cases. In the IIM mortalities not associated with infection, there were 2 acute myocardial infarction cases, 2 acute interstitial lung disease cases, 4 malignancies and 1 case of each of the following: Arrhythmia, pneumothorax, ventilator weakness, pulmonary artery hypertension, gastrointestinal bleeding, liver failure and renal failure. Three mortalities were secondary to viral hepatitis in the present study. Pathogenic infection was the most frequent cause of mortality in patients with IIM. The remaining causes
\end{abstract}

Correspondence to: Dr Hongjun Zhao or Dr Xiaoxia Zuo, Department of Rheumatology and Immunology, Xiangya Hospital, Central South University, 87 Xiangya Road, Changsha, Hunan 410008, P.R. China

E-mail: hongjunzhao2015@sina.com

E-mail: susanzuo@hotmail.com

Key words: idiopathic inflammatory myopathies, dermatomyositis, polymyositis of mortality included secondary to heart failure, lung dysfunction and malignancy. Following the ubiquitous application of glucocorticoids and immunosuppressants, the proportion of infection-associated mortalities increased in patients with IIM. Thus, in addition to focusing on the primary disease, infection should receive increased attention during clinical practice.

\section{Introduction}

Idiopathic inflammatory myopathies (IIM) are a group of non-suppurative inflammatory diseases that have been identified in skeletal muscle specimens. Although IIM are characterized by proximal muscle weakness, the etiology of IIM remains to be elucidated $(1,2)$. The incidence rate of IIM in the general population is high, with a range of 0.5-8.4 in every 100,000 individuals $(3,4)$.

Dermatomyositis (DM) and polymyositis (PM) are the most common IIM subtypes in clinical practice (2). The occurrence of these diseases often presents with the impairment of multiple systems, with a poor prognosis (1). It was previously reported that the mortality rate of patients with IIM was 2- to 3-fold higher than that of the normal population, and that infection, tumors and heart and lung impairment were the most common causes of mortality $(5,6)$. However, due to the large timespans reported in prior literature, in addition to differences in economic development and medical care in the areas described in those studies, significant differences exist in the mortality rate resulting from IIM (5-7).

The aim of the present study was to investigate and analyze the main causes of infection and of complications that have led to the mortality of patients with IIM in China since 2001.

\section{Materials and methods}

Review of patient information. The patients with IIM included in the current study adhered to the 1975 Bohan/Peter Diagnostic Standard (8). The current analyses were performed on 676 patients with IIM who were diagnosed in the Department of Rheumatism and Immunology at the Xiangya Hospital of Central South University from January, 2001 to January, 2015. The medical records of 49 patient mortalities (39 cases with DM and 10 cases with PM) were retrospectively 
analyzed. These data included information regarding clinical manifestations, laboratory examinations, treatments and clinical outcomes.

Analysis of the cause of mortality. The cause of mortality was determined following analysis of the patient medical records and discussion with the doctors responsible for a case. According to the clinical and pathological symptoms, the primary causes of mortality included infections, acute interstitial lung disease, ventilator weakness, pneumothorax, pulmonary artery hypertension, acute myocardial infarction, arrhythmia, gastrointestinal bleeding, liver failure, renal failure and tumors. The majority of mortalities were associated with infections and myositis. Infection-associated mortality was caused by pathogenic infections and by infection-associated complications resulting from IIM treatment. Myositis-associated mortality was due to multi-organ failure secondary to myositis and non-suppurative complications. If patients succumbed to infection during treatment, inflammatory-induced severe organ damage was considered to be an infection-associated mortality.

Statistical analysis. Data analyses were conducted using SPSS v. 18.0 (SPSS, Inc., Chicago, IL, USA). Continuous distribution variables are presented as mean \pm standard deviation. For equal measurement data, an independent samples t-test was used. Enumeration data were evaluated using a $\chi^{2}$ test. $\mathrm{P}<0.05$ was considered to indicate a statistically significant difference.

\section{Results}

Demographics. A total of 49 mortalities (7.2\%) resulted from 676 IIM cases, with mortality occurring following DM and PM in $6.9 \%(39 / 567)$ and $9.2 \%(10 / 109)$ of cases, respectively. No statistically significant difference was observed between the DM and PM mortality rates $\left(\chi^{2}=0.717 ; \mathrm{P}>0.05\right)$. Of the mortalities, 18 were male $(37.5 \%)$, after an average disease duration of $8.72 \pm 9.36$ months and at an average age of $48.83 \pm 12.19$ years. Of the female mortalities, the average disease duration was $10.09 \pm 9.02$ months, and the average age was $51.39 \pm 13.20$ years. No significant difference was observed between male and female in average disease duration or age $(\mathrm{t}=0.507 ; 0.671 ; \mathrm{P}>0.05)$.

Clinical manifestations. Of the 49 mortalities, the symptoms in 19 cases $(38.8 \%)$ began with skin lesions, while the symptoms of 17 cases $(34.7 \%$ ) began with myalgia and muscle weakness. The remainder of the patients (13 cases, $26.5 \%$ ) reported joint pain, coughing, gasping and edema as their initial symptoms. The majority of cases resulting in mortality developed complications: 33 patients who succumbed to the disease $(67.3 \%)$ had a lung infection, 28 patients $(57.1 \%)$ had an interstitial lung disease and 1 patient $(2.0 \%)$ presented pulmonary hypertension. Three patients $(6.1 \%)$ developed diabetes secondary to glucocorticoid treatments, and 4 patients (8.2\%) developed a decubitus ulcer due to being bedridden.

Treatment. Patients were administered $1 \mathrm{mg} / \mathrm{kg}$ prednisone daily. A number of immunosuppressants were administered to patients, as follows: Hydroxychloroquine (25/49 cases), mycophenolate mofetil (9/49 cases), thalidomide (27/49 cases), azathioprine (7/49 cases), methotrexate (10/49 cases) and cyclophosphamide (6/49 cases). Two patients with severe dysphagia were treated with intravenous immunoglobulin. Patients with an infection were administered anti-infective and symptom treatment.

Key causes of mortality. The cause of mortality in 49 patients, and their survival time, are shown in Table I. The most frequent causes of mortality were infection, heart and lung dysfunction and malignancy. The malignancy cases included breast cancer, lung cancer, nasopharyngeal carcinoma and ovarian cancer. Patients in which mortality was associated with an infection had a lower survival time than patients succumbing to myositis-associated mortality $(\mathrm{t}=-2.819 ; \mathrm{P}<0.05)$. In the cases leading to mortality, the proportion of patients with a survival time of $<1$ year due to an infection was higher than in patients succumbing to myositis-induced mortality $\left(\chi^{2}=6.110 ; \mathrm{P}<0.05\right)$.

The mortality rate of IIM patients admitted to the Xiangya Hospital was assessed in 3-year groupings from 2001 to 2015, together with the proportion of infection- or myositis-associated mortalities (Table II). The mortality rate decreased in the period of 2013-2015 compared with the previous time periods, but this was not identified as statistically significant $(\mathrm{P}>0.05)$. Although the proportion of infection-associated mortalities within the total number of patients with IIM was not significantly different among the time groupings, the proportion of mortalities secondary to organ failure due to myositis were significantly reduced over time $(\mathrm{P}<0.05)$. However, infection was the primary cause of mortality in patients with IIM. The proportion of infection-associated mortalities in patients with IIM increased, while the proportion of myositis-associated mortalities decreased (Fig. 1).

Infection sites and pathogens. In the 34 infection-associated mortality cases, the lungs were the primary site of infection, with the blood and intracalvarium as the second and third most common sites, respectively (Table III). Limb gangrene, an abdominal abscess, a decubitus ulcer on the buttocks and other foci of infection were also observed in a number of cases. Etiological diagnoses were conducted for 16 cases from lung, blood and urinary tract infections and a decubitus ulcer of the buttocks, based on sputum, blood, urine and secretion cultures and clinical manifestations. Intracranial infection was demonstrated by cerebrospinal fluid gram stain, revealing gram-positive cocci. Bacterial and fungal infections were identified in 14 and 3 cases, respectively. Co-infection with cytomegalovirus was also identified by serum antibody detection in 2 cases. Three viral hepatitis-associated mortalities were identified, with 2 of these being hepatitis B virus (HBV) cases and 1 being caused by hepatitis $\mathrm{C}$ virus (HCV).

\section{Discussion}

IIM is a form of autoimmune disease that is characterized by progressive muscle weakness of the symmetrical proximal limb muscles, periorbital edema, purple spots and a purple mound on the extensor side of the joint (2). IIM may also result in the damage of multiple organs. It has previously been 
Table I. Causes of mortality and survival time of 49 patients with idiopathic inflammatory myopathy.

\begin{tabular}{|c|c|c|c|c|}
\hline Cause of mortality & Cases & $\begin{array}{l}\text { Mean survival time } \\
\text { (months) }\end{array}$ & $\begin{array}{l}\text { Patients surviving } \\
<1 \text { year }\end{array}$ & $\begin{array}{c}\text { Patients surviving } \\
>1 \text { year }\end{array}$ \\
\hline Infection-associated & $34(69.4)$ & $7.3^{\mathrm{a}}$ & $26^{\mathrm{b}}$ & 8 \\
\hline Bacterial or fungal infection & $31(63.3)$ & 6.9 & 24 & 7 \\
\hline Viral hepatitis & $3(6.1)$ & 11.7 & 2 & 1 \\
\hline Myositis-associated & $15(30.6)$ & 14.7 & 6 & 9 \\
\hline Acute interstitial lung disease & $2(4.1)$ & 7.0 & 1 & 1 \\
\hline Ventilator weakness & $1(2.0)$ & 3.0 & 1 & 0 \\
\hline Pneumothorax & $1(2.0)$ & 3.0 & 1 & 0 \\
\hline Liver failure & $1(2.0)$ & 11.0 & 1 & 0 \\
\hline Pulmonary artery hypertension & $1(2.0)$ & 22.0 & 0 & 1 \\
\hline Acute myocardial infarction & $2(4.1)$ & 12.5 & 1 & 1 \\
\hline Arrhythmia & $1(2.0)$ & 21.0 & 0 & 1 \\
\hline Gastrointestinal bleeding & $1(2.0)$ & 13.0 & 0 & 1 \\
\hline Renal failure & $1(2.0)$ & 16.0 & 0 & 1 \\
\hline Tumor & $4(8.2)$ & 23.3 & 1 & 3 \\
\hline
\end{tabular}

Data are presented as $\mathrm{n}(\%)$ or $\mathrm{n}$. The survival time was significantly lower in cases of mortality associated with infection vs. mortality associated with myositis $\left({ }^{\mathrm{a}} \mathrm{P}<0.05\right)$, as evaluated using $\mathrm{t}$-tests $(\mathrm{t}=-2.819)$. The number of patients surviving $<1$ year was higher in the infection-associated group than the myositis-associated group ( $\left.{ }^{\mathrm{P}} \mathrm{P}<0.05\right)$, evaluated using Pearson's $\chi^{2}$ test $\left(\chi^{2}=6.110\right)$.

Table II. Rates of mortality caused by infection and myositis in idiopathic inflammatory myopathy patients, reported by year.

\begin{tabular}{|c|c|c|c|c|c|c|c|c|}
\hline \multirow[b]{2}{*}{ Cause of mortality } & \multicolumn{5}{|c|}{ Cases } & \multirow[b]{2}{*}{ Subtotal } & \multirow[b]{2}{*}{$\chi^{2}$} & \multirow[b]{2}{*}{ P-value } \\
\hline & 2001-2003 & 2004-2006 & 2007-2009 & 2010-2012 & 2013-2015 & & & \\
\hline Infection-associated & $3(4.4)$ & $3(3.1)$ & $5(4.2)$ & $9(6.0)$ & $14(5.8)^{\mathrm{a}}$ & $34(5.0)$ & 1.053 & 0.305 \\
\hline Myositis-associated & $3(4.4)$ & $4(4.1)$ & $3(2.5)$ & $2(1.3)$ & $3(1.2)$ & $15(2.2)$ & 4.339 & 0.037 \\
\hline Subtotal & $6(8.8)$ & $7(7.1)$ & $8(6.8)$ & $11(7.3)$ & $17(7.1)$ & $49(7.2)$ & 0.101 & 0.750 \\
\hline Total hospitalizations & 68 & 98 & 118 & 151 & 241 & 676 & & \\
\hline
\end{tabular}

Data are presented as $\mathrm{n}\left(\%\right.$ of total hospitalizations) or $\mathrm{n} .{ }^{\mathrm{a}} \mathrm{P}<0.05$, infection-associated mortality increased, but myositis-associated mortality $\operatorname{did} \operatorname{not}\left(\chi^{2}=4.755\right)$.

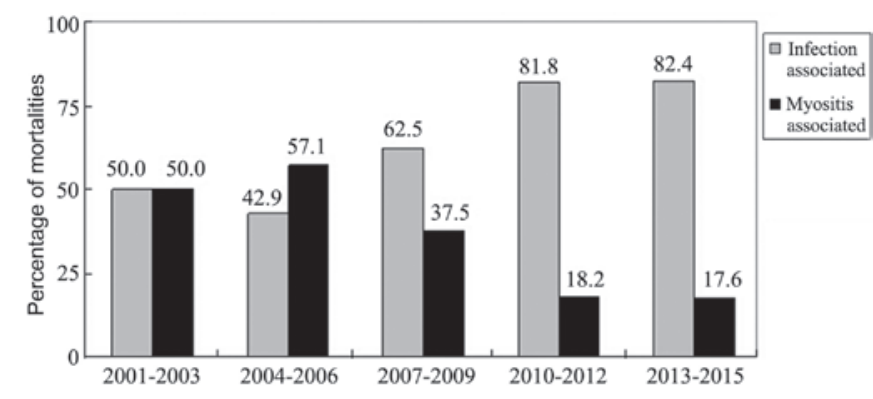

Figure 1. Alteration to ratio of causes of mortality in idiopathic inflammatory myopathy over time.

reported that although the administration of glucocorticoids and immunosuppressants decreases the risk of mortality from systemic lupus erythematosus, it may increase the risk of infections and associated complications (9). The present study showed that the proportion of infection-associated mortalities increased while the proportion of myositis-associated mortalities decreased in patients with IIM following treatment with glucocorticoids and immunosuppressants.

Infection is considered to be the primary cause of mortality in IIM patients. Based on the analysis of 15,407 patients with DM/PM from the HCUP Nationwide Inpatient Sample, Murray et al (10) reported that the mortality rate was significantly higher in patients with IIM who also had an infection. In the current study, the lungs and the blood were the primary infection sites, and bacteria and fungi were the primary pathogens. Patients with IIM with a pathogenic co-infection typically demonstrated a number of the following characteristics: Long-term glucocorticoid and immunosuppressant treatments; also suffering from diabetes, chronic viral hepatitis or kidney disease; interstitial lung disease causing pulmonary infection; damage in respiratory muscles, leading to increased difficulty removing secretions and resulting in ventilatory disorders; damage in the gastrointestinal muscles, resulting 
Table III. Infection sites and identifiable pathogens present in cases of infection-associated mortality associated with idiopathic inflammatory myopathy.

\begin{tabular}{|c|c|c|c|}
\hline Infection site & Cases & Pathogen & Cases \\
\hline \multirow[t]{10}{*}{ Lung infection } & \multirow[t]{10}{*}{25} & Candida parapsilosis & 1 \\
\hline & & Candida glabrata & 1 \\
\hline & & Candida albicans + cytomegalovirus & 1 \\
\hline & & Streptococcus pneumoniae & 1 \\
\hline & & Klebsiella pneumoniae & 2 \\
\hline & & Acinetobacter baumannii & 2 \\
\hline & & Pseudomonas alcaligenes + Acinetobacter baumannii & 1 \\
\hline & & Enterobacter cloacae + Stenotrophomonas maltophilia & 1 \\
\hline & & Mycobacterium tuberculosis & 1 \\
\hline & & Acinetobacter baumannii + cytomegalovirus & 1 \\
\hline Urinary tract infections & 3 & Escherichia coli & 1 \\
\hline Intracranial infection & 1 & Gram-positive cocci & 1 \\
\hline \multirow[t]{2}{*}{ Bloodstream infection } & \multirow[t]{2}{*}{6} & Methicillin-resistant Staphylococcus aureus & 1 \\
\hline & & Proteus mirabilis & 1 \\
\hline Right upper limb gangrene & 1 & & \\
\hline Abdominal abscess & 1 & & \\
\hline Decubitus ulcer of the buttocks & 3 & Pseudomonas aeruginosa & 1 \\
\hline
\end{tabular}

in dysphagia and pulmonary aspiration defects; and decubitus ulcers and delayed healing, secondary to nutritional deficiencies and being bedridden. The most common bacteria causing infections in IIM cases included Klebsiella pneumoniae, Acinetobacter baumannii, Streptococcus pneumoniae, Staphylococcus aureus and Candida spp. Co-infections, opportunistic infections, invasive fungal infections and viral infections were common. During IIM therapy, pneumococcal and influenza vaccinations should be administered for prevention (1). Additionally, glucocorticoid dosages should be gradually reduced when the condition is controlled.

It can be concluded from the present study that physicians should consider HBV and HCV infections in patients with IIM as long-term glucocorticoid and immunosuppressant treatments that may cause the reactivation of viral hepatitis. In the current study, 2 patients were HBV surface antigen-positive, HBV antibody e-positive and hepatitis $\mathrm{B}$ core antibody-positive following diagnosis with IIM and 1 patient was anti-HCV antibody-positive but all 3 patients demonstrated normal liver function. However, following glucocorticoid and methotrexate administration, viral replication was reactivated in the patients presenting with viral resistance to the antiviral therapy. The levels of aspartate aminotransferase and alanine aminotransferase in these patients was elevated and they were jaundiced, with an outcome of liver failure and mortality in all 3 cases. Screening for HBV and $\mathrm{HCV}$ should therefore be conducted prior to the initiation of IIM therapy. If patients with IIM are positive for HBV and HCV, anti-viral therapy (such as entecavir or tenofovir disoproxil) should be administered 2-4 weeks prior to immunosuppressive therapy and until 6 months after the immunosuppressive therapy has been ceased. This administration should occur even if HBV-DNA and HCV-RNA are not detectable and the liver enzyme level is normal in these patients. If anti-viral therapy is administered following liver enzyme elevation, recurrence of viral hepatitis may result in liver failure (11).
Impaired heart function was observed in 9-72\% of the IIM patients, predominantly presenting as sinus tachycardia and atrioventricular block, and a number of cases also presented with associated symptoms (12). In the present study, 1 patient succumbed to complete heart failure, and 2 patients succumbed to heart failure induced by an acute myocardial infarction. Impaired lung function secondary to IIM typically presented as interstitial lung disease, aspiration pneumonia and hypoventilation. Previous studies indicated that interstitial lung disease may occur in $36-65 \%$ of patients with IIM (13). In the present study, 1 patient succumbed to right-side heart failure that was associated with pulmonary hypertension resulting from interstitial lung disease. Pulmonary hypertension may occur in $5.2 \%$ of IIM patients and reduce the quality of life of patients (14). Without treatment, pulmonary hypertension in IIM may cause right-side heart failure, arrhythmia and sudden mortality (15). Furthermore, a previous study of 150 patients with IIM suggested that impaired renal function occurred in $23.3 \%$ of patients, and that 10.7 and $20.7 \%$ had acute kidney disease and chronic kidney disease, respectively (16). Acute kidney disease predominantly resulted from drug- and myoglobin-induced renal papillary necrosis; $12.5 \%$ of patients then progressed to end-stage renal disease (17). In the present study, 1 patient succumbed to renal disease due to hemodialysis cessation. However, as therapy for renal function replacement was widely used, few patients succumbed to chronic renal failure. Gastrointestinal bleeding is rare in patients with IIM, and only few cases have been reported worldwide, but visceral vasculitis may be a potential cause (18-20). A number of reports have indicated that intravenous immunoglobulin may be an effective treatment for patients with IIM with severe gastrointestinal disease (21). Furthermore, patients with IIM presenting with a skin rash should be mindful of the risk of malignant atrophic papulosis due to the subsequent poor prognosis, even with passive therapy (22). 
Previous studies have reported that the prognosis of IIM may be affected by gender, age, proximal muscle weakness, dysphagia, hypoventilation, interstitial lung disease, serum creatine kinase level and delayed diagnosis or treatment (23). Glucocorticoids remain the primary choice in treating PM and DM. A number of clinicians immediately administer immunosuppressants following confirmation of an IIM diagnosis (24). However, there is a lack of large-scale supporting evidence for this approach, thus that doctors typically depend on their experience in the treatment of patients with IIM.

The limitations of the present study are the absence of postmortem autopsy, and its retrospective nature as it is difficult to differentiate between DM/PM and IBM without an autopsy. Causes of these mortalities may therefore be substantially different from those succumbing to this disease outside of a hospital.

The mortality rate of patients with IIM has decreased in recent years, but is primarily associated with infection. In addition to the occurrence of bacterial infections, viral hepatitis necessitates increased consideration. Although myositis-associated mortality and concurrent complications decreased over time, infection-associated mortality increased. As a result, the present study concludes that infection in IIM cases necessitates increased focus to decrease the mortality rate.

\section{References}

1. Ernste FC and Reed AM: Idiopathic inflammatory myopathies: Current trends in pathogenesis, clinical features, and up-to-date treatment recommendations. Mayo Clin Proc 88: 83-105, 2013.

2. Rider LG and Miller FW: Deciphering the clinical presentations, pathogenesis, and treatment of the idiopathic inflammatory myopathies. JAMA 305: 183-190, 2011.

3. Furst DE, Amato AA, Iorga SR, Gajria K and Fernandes AW: Epidemiology of adult idiopathic inflammatory myopathies in a U.S. managed care plan. Muscle Nerve 45: 676-683, 2012.

4. Oddis CV, Conte CG, Steen VD and Medsger TA Jr: Incidence of polymyositis-dermatomyositis: A 20-year study of hospital diagnosed cases in Allegheny County, PA 1963-1982. J Rheumatol 17: 1329-1334, 1990.

5. Woo JH, Kim YJ, Kim JJ, Choi CB, Sung YK, Kim TH, Jun JB, Bae SC and Yoo DH: Mortality factors in idiopathic inflammatory myopathy: Focusing on malignancy and interstitial lung disease. Mod Rheumatol 23: 503-508, 2013.

6. Maldonado F, Patel RR, Iyer VN, Yi ES and Ryu JH: Are respiratory complications common causes of death in inflammatory myopathies? An autopsy study. Respirology 17: 455-460, 2012.

7. Torres C, Belmonte R, Carmona L, Gómez-Reino FJ, Galindo M, Ramos B, Cabello A and Carreira PE: Survival, mortality and causes of death in inflammatory myopathies. Autoimmunity 39: 205-215, 2006
8. Bohan A and Peter JB: Polymyositis and dermatomyositis (first of two parts). N Engl J Med 292: 344-347, 1975.

9. Fei Y, Shi X, Gan F, Li X, Zhang W, Li M, Hou Y, Zhang X, Zhao Y, Zeng X and Zhang F: Death causes and pathogens analysis of systemic lupus erythematosus during the past 26 years. Clin Rheumatol 33: 57-63, 2014.

10. Murray SG, Schmajuk G, Trupin L, Lawson E, Cascino M, Barton J, Margaretten M, Katz PP, Yelin EH and Yazdany J: A population-based study of infection-related hospital mortality in patients with dermatomyositis/polymyositis. Arthritis Care Res 67: 673-680, 2015.

11. Martin P, Lau DT, Nguyen MH, Janssen HL, Dieterich DT, Peters MG and Jacobson IM: A treatment algorithm for the management of chronic hepatitis B virus infection in the United States: 2015 update. Clin Gastroenterol Hepatol 13: 2071-2087, 2015.

12. Van Gelder H and Charles-Schoeman C: The heart in inflammatory myopathies. Rheum Dis Clin North Am 40: 1-10, 2014.

13. Fathi M, Lundberg IE and Tornling G: Pulmonary complications of polymyositis and dermatomyositis. Semin Respir Crit Care Med 28: 451-458, 2007.

14. Zhang L, Wang GC, Ma L and Zu N: Cardiac involvement in adult polymyositis or dermatomyositis: A systematic review. Clin Cardiol 35: 686-691, 2012.

15. Minai OA: Pulmonary hypertension in polymyositis-dermatomyositis: Clinical and hemodynamic characteristics and response to vasoactive therapy. Lupus 18: 1006-1010, 2009.

16. Couvrat-Desvergnes G, Masseau A, Benveniste O, Bruel A, Hervier B, Mussini JM, Buob D, Hachulla E, Rémy P, Azar R, et al: The spectrum of renal involvement in patients with inflammatory myopathies. Medicine (Baltimore) 93: 33-41, 2014.

17. Qian Y, Ren H, Chen X, Zhang W, Li X, Shi H, Chen N: Renal injury in patients with polymyositis and dermatomyositis. Shang Hai Jiao Tong Da Xue 31: 451-454, 2011 (In Chinese).

18. Chen GY, Liu MF, Lee JY and Chen W: Combination of massive mucinosis, dermatomyositis, pyoderma gangrenosum-like ulcer, bullae and fatal intestinal vasculopathy in a young female. Eur J Dermatol 15: 396-400, 2005

19. Marie I, Levesque H, Cailleux N, Courtois H and Guédon C: Intravenous immunoglobulin for treatment of gastro-intestinal haemorrhage in dermatomyositis. Ann Rheum Dis 60: 723-724, 2001.

20. Tweezer-Zaks N, Ben-Horin S, Schiby G, Bank I, Levi Y, Livneh A and Langevitz P: Severe gastrointestinal inflammation in adult dermatomyositis: Characterization of a novel clinical association. Am J Med Sci 332: 308-313, 2006.

21. Wang DX, Shu XM, Tian XL, Chen F, Zu N, Ma L and Wang GC: Intravenous immunoglobulin therapy in adult patients with polymyositis/dermatomyositis: A systematic literature review. Clin Rheumatol 31: 801-806, 2012.

22. Burgin S, Stone JH, Shenoy-Bhangle AS and McGuone D: Case records of the Massachusetts General Hospital. Case 18-2014. A 32-year-old man with a rash, myalgia, and weakness. N Engl J Med 370: 2327-2337, 2014.

23. Brandão M and Marinho A: Idiopathic inflammatory myopathies: Definition and management of refractory disease. Autoimmun Rev 10: 720-724, 2011.

24. Carstens PO and Schmidt J: Diagnosis, pathogenesis and treatment of myositis: Recent advances. Clin Exp Immunol 175: 349-358, 2014. 